\title{
Vectors and Beyond: Geometric Algebra and its Philosophical Significance
}

Peter Simons

In mathematics, like in everything else, it is the Darwinian struggle for life of ideas that leads to the survival of the concepts which we actually use, often believed to have come to us fully armed with goodness from some mysterious Platonic repository of truths.

Simon Altmann

\section{Introduction}

The purpose of this paper is to draw the attention of philosophers and others interested in the applicability of mathematics to a quiet revolution that is taking place around the theory of vectors and their application. It is not that new mathematics is being invented - on the contrary, the basic concepts are over a century old - but rather that this old theory, having languished for many decades as a quaint backwater, is being rediscovered and properly applied for the first time. The philosophical importance of this quiet revolution is not that new applications for old mathematics are being found. That presumably happens much of the time. Rather it is that this new range of applications affords us a novel insight into the reasons why vectors and their mathematical kin find application at all in the real world. Indirectly, it tells us something general but interesting about the nature of the spatiotemporal world we all inhabit, and that is of philosophical significance. Quite what this significance amounts to is not yet clear. I should stress that nothing here is original: the history is quite accessible from several sources, and the mathematics is commonplace to those who know it. However, for philosophers who are not themselves mathematicians, I hope this may be of some interest.

\section{Excerpts from the history of vector theory}

\section{Dramatis personae}

Benjamin Olinde Rodrigues (1795-1851)

Sir William Rowan Hamilton (1805-1865)

Hermann Günther Grassmann (1808-1877)

Peter Guthrie Tait (1831-1901)

$\dagger$ Department of Philosophy, Trinity College Dublin, Dublin 2, Ireland; Email:
psimons@tcd.ie 
Josiah Willard Gibbs (1839-1903)

William Kingdon Clifford (1845-1879)

Oliver Heaviside (1850-1925)

David Hestenes (b. 1933)

There is a classic history of vector theory, a marvellous book by Michael Crowe (1967). The subsequent history since Crowe's book appeared shows that while he is quite right that vectors won out over quaternions in "the Great Quaternionic War" of roughly 1890-1910, it is no longer the case, as it seemed to him then, that vector analysis plus other things will remain the undisputed formalism of choice in modern applied mathematics.

Although the idea of directed quantities was present in earlier mathematics, it emerged most obviously in the Wessel-Argand geometric interpretation of complex numbers. It was after thirteen years of trying to generalize this to three dimensions that in a famous episode Hamilton got the insight that three rather than two extra imaginary units were needed, and this was the key to the quaternions. The discovery, which can be located in space and time to a very small region near Broome Bridge in Dublin on the morning of 16 October 1843, is one of the best-known stories of mathematics.

Quaternions (sometimes called 'hypercomplex numbers') are quantities:

$$
q=w+\mathbf{i} x+\mathbf{j} y+\mathbf{k} z
$$

where $w, x, y, w$ are real, addition is defined termwise, and the imaginary units i j k satisfy:

$$
\mathbf{i}^{2}=\mathbf{j}^{2}=\mathbf{k}^{2}=\mathbf{i j k}=-1 ; \mathbf{i j}=-\mathbf{j i}=\mathbf{k} ; \mathbf{j k}=-\mathbf{k j}=\mathbf{i} ; \mathbf{k} \mathbf{i}=-\mathbf{i k}=\mathbf{j}
$$

Quaternions form the largest (non-commutative) division algebra: every non-zero quaternion has an inverse. From 1846 Hamilton called $w$ the scalar part and $\mathbf{i} x+\mathbf{j} y+\mathbf{k} z$ the pure or vector part of a quaternion, and if $q, q^{\prime}$ are two pure quaternions (vectors):

$$
q q^{\prime}=-\left(x x^{\prime}+y y^{\prime}+z z^{\prime}\right)+\mathbf{i}\left(y z^{\prime}-z y^{\prime}\right)+\mathbf{j}\left(z x^{\prime}-x z^{\prime}\right)+\mathbf{k}\left(x y^{\prime}-y x^{\prime}\right)
$$

So quaternion multiplication embodies both (the negative of) the scalar product, and the vector product of later theory.

Just as complex numbers handle rotations in the plane, so quaternions are meant to handle rotations in three-dimensional space. But, misled by the analogy with the two-dimensional case, where multiplication by a single unit complex number produces a rotation, Hamilton assumed such rotations could be represented by a single multiplication $q \mathbf{v}$, where $\mathbf{v}$ is any vector and $q$ is a unit quaternion. This one-sided multiplication works only for a special case: rotations orthogonal to the 
vector, about its axis. Thus from the very evening of 16 October 1843 Hamilton bungled the quaternion account of rotation, which should be dealt with by pre- and postmultiplication by a quaternion $r \mathbf{v} r^{-1}$, with $r$ defined not (as in Hamilton) by the angle of the rotation but by its half-angle. The geometry of composing rotations and the need to deal in half-angles had already been properly done in 1840 by a French mathematician who was also a socialist banker, Benjamin Olinde Rodrigues, but had (not unsurprisingly) escaped Hamilton's notice. Particularly insightful and detailed accounts of Hamilton's mistake and Rodrigues's success are given by Altmann $(1986,1989,1992)$, who deserves much credit for showing that the neglect of quaternions was for a long time excessive, as well as for investigating the hitherto highly obscure Rodrigues (Altmann and Ortiz, 2005). ${ }^{1}$

Hamilton was convinced that quaternions were the key to the universe. For a while, spurred by his deserved fame and his enthusiastic advocacy, quaternions were all the rage. His 1853 Lectures on Quaternions ran to nearly 900 pages, and James Clerk Maxwell used them though without total endorsement in his Treatise on Electricity and Magnetism. In the propagation of the quaternionic gospel, Hamilton was ably seconded by his bulldog, the Scottish mathematician Peter Guthrie Tait, but gradually the drawbacks of quaternions became apparent, not least through the work of Maxwell, who eventually dropped them. Quaternions came under fire from two thinkers who independently came up with the more flexible mathematics of vectors, the American mathematician-physicist-chemist Josiah Willard Gibbs and the English engineer-physicist Oliver Heaviside. Gibbs's Yale lectures on vector analysis began to be circulated around 1881, while Heaviside's Electromagnetic Theory came out in 1891. After the publication in 1901 by Edwin Bidwell Wilson of Vector Analysis: A Text Book for the Use of Students of Mathematics and Physics and Founded upon the Lectures of J. Willard Gibbs, the war was decided. That book reads like any modern textbook of vector theory, and indeed it was the first such. For example the definitions of scalar and vector are wholly modern:

Definition: A vector is a quantity which is considered as possessing direction as well as magnitude.

Definition: A scalar is a quantity which is considered as possessing magnitude but no direction.

Gibbs and Heaviside arrived at their ideas independently and in a remarkably similar way: by separating the scalar and vector parts of the quaternion product as two distinct products, and changing the sign of the scalar part. After embarking on vector theory, Gibbs discovered some convergence with ideas that had already

${ }^{1}$ Quarternions have, since Altmann published his book in 1986, and in part because of the book, had about as much of a revival as they merit: they are now regularly used by space engineers and computer games developers for efficient and singularity-free representations of 3-dimensional spatial rotations, as of space vehicles and virtual game perspectives. See Kuipers (2002). 
been proposed by Hermann Günther Grassmann. ${ }^{2}$ Grassmann was, in his day, and even to some extent now, one of the most unjustly neglected mathematicians of all time. His revolutionary Ausdehnungslehre [Theory of Extension] was first published in 1844 (Grassmann 1844), with a second revised and cut-down edition in 1862 (Grassmann 1862) and a third, which restored some of the first edition's cuts, appearing in 1878 only after his death. Grassmann was led to his algebra of geometry initially by a problem in applied mathematics: that of describing the flow of water in tides (Grassmann 1840). For this purpose he introduced two notions which later became known as the scalar (inner) product and exterior (or outer) product of two vectors. Grassmann's work, which was conceived for any finite number of dimensions, not just two or three, was more revolutionary than Hamilton's and at a level of abstraction which even professional mathematicians such as Möbius and Kummer found offputting. The Gibbs-Heaviside vector theory is as much a cut-down version of Grassmann's work as of Hamilton's, though because of Grassmann's obscurity they discovered that only subsequently. Vectors in the Gibbs-Heaviside vein caught on rapidly in the early twentieth century, being both adequate to many applications and easy enough for non-mathematicians to grasp and manipulate. From then on, vector algebra and vector analysis have held sway throughout most of the twentieth century.

The quaternionists did not however give up without a massive struggle. In 1888 Gibbs wrote in a letter:

a Kampf ums Dasein is just commencing between the different methods and notations of multiple algebra, especially between the ideas of Grassmann \& of Hamilton.

The rhetoric in the Great Quaternionic War became quite heated. So Tait wrote:

Even Professor Gibbs must be ranked as one of the retarders of Quaternion progress, in virtue of his pamphlet on Vector Analysis, a sort of hermaphrodite monster, compounded of the notations of Hamilton and of Grassmann.

Heaviside responded with eponymous irony:

the invention of quaternions must be regarded as a most remarkable feat of human ingenuity. Vector analysis, without quaternions, could have been found by any mathematician by carefully examining the mechanics of the Cartesian mathematics; but to find out quaternions required a genius.

Lord Kelvin in 1892 dismissed quaternions in no uncertain terms:

Quaternions came from Hamilton after his really good work had been done; and, though beautifully ingenious, have been an unmixed evil to those who have touched them in any way, including Clerk Maxwell.

2 Grassmann wrote his own surname 'Graßmann' but even German writers do not uniformly spell it this way, and it appeared as 'Grassmann' on the title page of the 1844 Ausdehnungslehre, so we follow the general practice. For his collected works see Grassmann (1894-1911). For the English translation of the Ausdehnungslehre, see Grassmann (1995). 
The heat prompted another physical peer, Lord Rayleigh, to quip in echo of Tertullian, "Behold how these vectorists love one another". 3

One irony of subsequent developments was that while Gibbs drew some inspiration from Grassmann, the success of vector analysis sidelined Grassmann as much as it did Hamilton when it came to applications, without Grassmann even having had the benefit of being once on the cutting edge of fashion. As vectors came into more general use in physics and other applications, the march of developments meant however that vector analysis needed to be supplemented by a congeries of other mathematical techniques: tensors, spinors, matrix algebra, Hilbert spaces, differential forms, and more. The result is a bewildering plethora of mathematical techniques which require much learning and teaching, which tend to fragment the subject and which embody wasteful overlaps and requirements of translation.

\section{The first geometric algebra}

The true father of geometric algebra was one of those very few mathematicians who were able to appreciate and combine insights from both Hamilton and Grassmann, namely William Kingdon Clifford (1845-1879). ${ }^{4}$ Clifford was a Victorian polymath of that sort whose breadth, optimism and restless energy made their age so fertile and in some ways so alien to ours. His wonderfully lucid and accessible The Common Sense of the Exact Sciences, unfinished at his early death, completed and published posthumously by Karl Pearson, inspired the young Bertrand Russell. Clifford's energy was his undoing: he seems to have literally worn himself out by the age of 33. In his 1878 Elements of Dynamic (Clifford 1878 and 1887) he modified Grassmann's theory, developing the geometric product as the sum of the scalar and outer products:

$$
\mathbf{a b}=\mathbf{a} \cdot \mathbf{b}+\mathbf{a} \wedge \mathbf{b}
$$

This combines the scalar (inner) product $\mathbf{a} \cdot \mathbf{b}$ and the vector (outer) product $\mathbf{a} \wedge \mathbf{b}$ (read 'a wedge $\mathbf{b}$ ') of his predecessors, in a new way whose significance we shall see. ${ }^{5}$ The outer product of two vectors is a new kind of object called a bivector. Initially the decision to combine the two looks arbitrary or capricious, though there are precedents in both Hamilton and Grassmann. In fact since the scalar product is symmetric $(\mathbf{a} \cdot \mathbf{b}=\mathbf{b} \cdot \mathbf{a})$ while the outer product is skew-symmetric (1898)

${ }^{3}$ All quotes are taken from Crowe (1967).

${ }^{4}$ See Clifford $(1878,1882)$. Another was Alfred North Whitehead: see Whitehead

${ }^{5}$ Clifford's treatment differs from Grassmann's and Hamilton's on a seemingly minor algebraic point. Where $\mathbf{e}_{i}$ and $\mathbf{e}_{j}$ are orthogonal units, all three have $\mathbf{e}_{i} \mathbf{e}_{j}=-\mathbf{e}_{j} \mathbf{e}_{i}$ when $i \neq j$; Grassmann has $\mathbf{e}_{i}^{2}=0$, Hamilton has $\mathbf{e}_{i}^{2}=-1$, and Clifford has $\mathbf{e}_{i}^{2}=1$. That turns out to make all the difference. 
$(\mathbf{a} \wedge \mathbf{b}=-\mathbf{b} \wedge \mathbf{a})$, the two parts of the geometric product of two vectors represent the symmetric and skew-symmetric parts respectively of that product. Indeed to partly sidestep the awkward question as to how a scalar can be added to a bivector, it is theoretically preferable to treat the geometric product as primitive and governed by axioms, and define the scalar and outer products respectively as:

$$
\begin{aligned}
& \mathbf{a} \cdot \mathbf{b}={ }_{\text {Df. }} 1 / 2(\mathbf{a b}+\mathbf{b a}) \\
& \mathbf{a} \wedge \mathbf{b}={ }_{\text {Df. }} 1 / 2(\mathbf{a b}-\mathbf{b a})
\end{aligned}
$$

\section{Some basic notions of geometric algebra}

To motivate some of the philosophical remarks later it is useful to have a smattering of the basics of geometric algebra. This section is just to give such a flavour: it is not intended as a proper introduction, for which see the works by Hestenes, Doran and Lasenby given in the references.

Let's start with two dimensions. Suppose $\mathbf{e}_{\mathbf{1}}$ and $\mathbf{e}_{2}$ to be two orthonormal vectors, so $\left|\mathbf{e}_{1}\right|=\left|\mathbf{e}_{2}\right|=1$, and let $\mathbf{a}$ and $\mathbf{b}$ be two vectors with:

$$
\begin{aligned}
& \mathbf{a}=a_{1} \mathbf{e}_{\mathbf{1}}+a_{2} \mathbf{e}_{\mathbf{2}} \\
& \mathbf{b}=b_{1} \mathbf{e}_{\mathbf{1}}+b_{2} \mathbf{e}_{2}
\end{aligned}
$$

where the $a_{i}$ and $b_{j}$ are, as usual, real scalars (real numbers). The sum of these two vectors $\mathbf{a}+\mathbf{b}$ is computed coordinatewise as usual:

$$
\mathbf{a}+\mathbf{b}=\left(a_{1}+b_{1}\right) \mathbf{e}_{1}+\left(a_{2}+b_{2}\right) \mathbf{e}_{2}
$$

We look now for an associative, distributive (over + ) product of vectors such that any vector squares to the square of its magnitude: $\mathbf{a}^{2}=|\mathbf{a}|^{2}=a_{1}{ }^{2}+a_{2}{ }^{2}$. To fulfil these conditions, in particular the last one, we have:

$$
\begin{aligned}
\mathbf{a}^{2} & =\left(a_{1} \mathbf{e}_{1}+a_{2} \mathbf{e}_{2}\right)\left(a_{1} \mathbf{e}_{1}+a_{2} \mathbf{e}_{2}\right) \\
& =a_{1}{ }^{2}+a_{2}{ }^{2}+a_{1} a_{2}\left(\mathbf{e}_{1} \mathbf{e}_{2}+\mathbf{e}_{2} \mathbf{e}_{1}\right)
\end{aligned}
$$

So $\mathbf{e}_{1} \mathbf{e}_{2}+\mathbf{e}_{2} \mathbf{e}_{1}=0$

and $\mathbf{e}_{1} \mathbf{e}_{2}=-\mathbf{e}_{2} \mathbf{e}_{1}$

so $\left(\mathbf{e}_{1} \mathbf{e}_{2}\right)^{2}=\left(\mathbf{e}_{1} \mathbf{e}_{2}\right)\left(\mathbf{e}_{1} \mathbf{e}_{2}\right)=\mathbf{e}_{1}\left(\mathbf{e}_{2} \mathbf{e}_{1}\right) \mathbf{e}_{2}=-\mathbf{e}_{1}^{2} \mathbf{e}_{2}^{2}=-1$

This product $\mathbf{e}_{1} \mathbf{e}_{2}$ is neither a scalar nor a vector but a third kind of object, a bivector. Notice that its geometric square (the geometric product of itself with itself) is -1 , a feature we normally associate only with the complex units $\pm i$. 
It is hard to overestimate the importance of bringing in bivectors alongside vectors and scalars. As a vector may be geometrically associated with a directed line segment (normally abstracting from its point of origin, so invariant under parallel translation), the length of the line corresponding to the vector's magnitude, its direction and orientation to those of the line, so a bivector may be associated geometrically with an oriented planar region, the area of the region corresponding to the bivector's magnitude, the plane it is in being its twodimensional "direction", and the orientation of the region being the "handedness" of the bivector. The area associated with the bivector $\mathbf{a} \wedge \mathbf{b}$ is that of any parallelogram based on $\mathbf{a}$ and $\mathbf{b}$, and its "handedness" is given by tracing the vectors, first $\mathbf{a}$, then $\mathbf{b}$ :

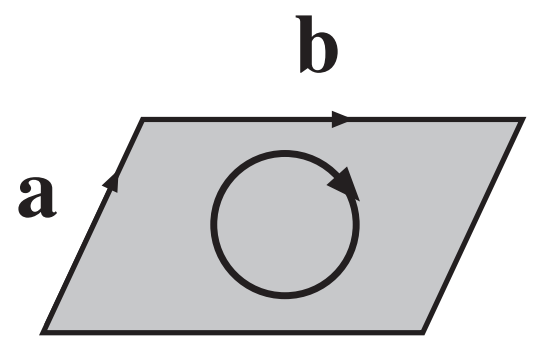

Obviously the opposite order $\mathbf{b}$ then a will produce a parallelogram with the same area but opposite orientation,

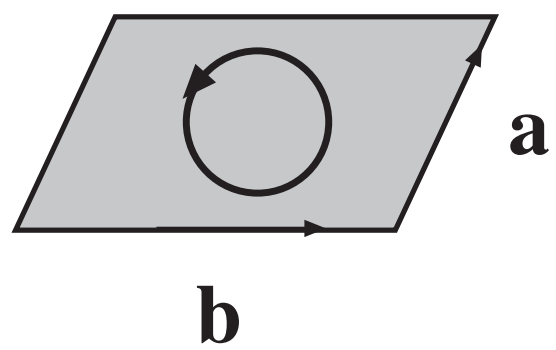

while the parallelogram of two orthonormal vectors is a square of area 1 .

We now extend this collection of geometrical objects by adding not only scalars and bivectors but also vectors to the mix. The result is a Clifford Algebra.

The Clifford algebra $\boldsymbol{C l}_{2}$ of 2-dimensional space is a 4-dimensional collection of elements:

$$
A=a_{0}+a_{1} \mathbf{e}_{1}+a_{2} \mathbf{e}_{2}+a_{12} \mathbf{e}_{12}
$$

where the $a_{k}$ are real, addition and multiplication are defined elementwise, and $\mathbf{e}_{12}=\mathbf{e}_{1} \mathbf{e}_{2}$. The Clifford algebra is spanned by the four base elements $1, \mathbf{e}_{1}, \mathbf{e}_{2}, \mathbf{e}_{12}$ 
and its parts are of grade 0 (scalars), 1 (vectors) and 2 (bivectors). A sum of elements of different grades is called a multivector or blade. Like quaternions and complex numbers, Clifford algebras admit of division. Unlike these, they can be of arbitrary finite dimension.

In three dimensions, in addition to scalars, vectors and bivectors, there are products of three vectors not reducible to the others, trivectors. The Clifford algebra $\mathrm{Cl}_{3}$ of 3-dimensional space is an 8-dimensional linear collection of blades:

$$
A=a_{0}+a_{1} \mathbf{e}_{1}+a_{2} \mathbf{e}_{2}+a_{3} \mathbf{e}_{3}+a_{12} \mathbf{e}_{12}+a_{23} \mathbf{e}_{23}+a_{31} \mathbf{e}_{31}+a_{123} \mathbf{e}_{123}
$$

with a basis of 1 scalar, 3 vectors, 3 bivectors and 1 trivector, where the trivector represents an oriented unit volume. It contains subalgebras isomorphic to both the complex numbers and the quaternions. The unit trivector $\mathbf{e}_{123}=\mathbf{e}_{1} \mathbf{e}_{2} \mathbf{e}_{3}$ squares to -1 , and it is quite common to notate it as $I$, since it has the same multiplication property as the imaginary unit. In general the highest-dimension elements of a Clifford algebra of a given dimension are called pseudoscalars and can be expressed as scalar multiples $\beta I$ of the unit pseudoscalar $I$ (here a unit trivector). So a general element of $\boldsymbol{C l}_{3}$ is a sum:

$$
A=\alpha+\mathbf{a}+B+\beta I
$$

where $\alpha$ and $\beta$ are scalars, $\mathbf{a}$ is a vector, and $B$ is a bivector.

An important operation in Clifford algebra is reversion, which is obtained by reversing the order of all geometric products of vectors. Reversion leaves scalars and vectors invariant but switches the sign of bivectors and pseudoscalars:

$$
A \dagger=\alpha+\mathbf{a}-B-\beta I
$$

It is thus computationally akin to the complex conjugate among numbers and the Hermitian operator among matrices.

The bivector $\mathbf{a} \wedge \mathbf{b}$ replaces the standard vector product $\mathbf{a} \times \mathbf{b}$, which is a vector orthogonal to $\mathbf{a}$ and $\mathbf{b}$, not a bivector. The cross product is only definable in three dimensions but the wedge product is definable in any number of dimensions. In three-dimensional space the cross product is the Clifford dual of the wedge product. The importance of having an intrinsically defined bivector instead of a dual vector is twofold. Firstly it obviates an unfortunate and confusing duality in the application of vectors, namely the distinction between polar vectors (which are invariant under reflection parallel to the vector but reverse under orthogonal reflection) and axial vectors (which are the other way round) ${ }^{6}$ Secondly, there are theoretically some quantities such as angular momentum of a spinning planar

${ }^{6}$ Cf. Altmann (1992). 
object where the third dimension is or may be missing, so the quantity should be confined to the two dimensions of the plane. The bivector $\mathbf{a} \wedge \mathbf{b}$ is precisely in the plane itself: its orientation characterises the direction of spin and its magnitude the magnitude of the angular momentum.

A particularly important feature of Clifford algebras, which allows $\boldsymbol{C l}_{3}$ to subsume the quaternions as well as the complex numbers, is their treatment of rotations. Let $\mathbf{n}$ and $\mathbf{m}$ be any two non-parallel unit vectors, which therefore define a plane, that of $\mathbf{m} \wedge \mathbf{n}$. A rotation in the plane generated by $\mathbf{m}$ and $\mathbf{n}$ of any vector $\mathbf{a}$ is given by successive reflections in the hyperplanes perpendicular to $\mathbf{m}$ and $\mathbf{n}$. The component of a perpendicular to the $\mathbf{m} \wedge \mathbf{n}$ plane is unaffected, while by simple trigonometry the resultant vector is at an angle $2 \theta$ from a, where $\theta$ is the angle between $\mathbf{m}$ and $\mathbf{n}, \mathbf{m} \cdot \mathbf{n}=\cos (\theta)$. This is again Rodrigues' half-angle result. Using geometric algebra we get that the vector $\mathbf{a}$ is rotated into the vector $\mathbf{b}=\mathbf{n m a m n}$. Defining a unit bivector $R=\mathbf{n m}$ this means that we rotate $\mathbf{a}$ through twice the angle between $\mathbf{m}$ and $\mathbf{n}$ by operating on a using $R$ and its reverse $R \dagger$, so:

$$
\mathbf{b}=R \mathbf{a} R \dagger
$$

And we can add that $R=\mathbf{n m}=\mathbf{n} \cdot \mathbf{m}+\mathbf{n} \wedge \mathbf{m}=\cos (\theta)+\mathbf{n} \wedge \mathbf{m}$. The object $R$ is a sum of a scalar and a bivector, and its magnitude is unity, since $|\mathbf{n} \wedge \mathbf{m}|=\sin (\theta)$. It is called a rotor. In two dimensions rotations are handled by multiplying by unit complex numbers, in three dimensions they can be handled by pre- and postmultiplication by unit quaternions, but in Clifford algebra the construction works in any number of dimensions, and also works for any grade of multivector.

\section{The modern revival of Clifford algebra as geometric algebra}

Clifford's own name for his algebra was 'geometric algebra', which echoes the ideas, words and aspirations of Grassmann. The development of the algebra and analysis of geometrical ideas along Clifford's lines was, like the work of Hamilton and Grassmann, eclipsed by the success of vector theory. But vectors led to a wasteful and obscuring fragmentation of the mathematical treatment of geometrical notions. The rediscovery of Clifford's ideas, their exploitation and adaptation for the efficient representation of geometric notions is the achievement of the American mathematical physicist David Hestenes (b. 1933) (Hestenes 1966 and 1986; Hestenes and Sobczyk 1984). It was Hestenes who proposed returning to Clifford's own designation, which like him we abbreviate to 'GA'. To give a (not wholly unbiased) indication of the advantages of GA over other approaches we may quote Hestenes' list of features integrated by GA, as given in his 2002 Oersted Medal Lecture (Hestenes 2003), namely synthetic geometry, coordinate geometry, complex variables, quaternions, vector analysis, matrix algebras, spinors, tensors, and differential forms. Since few physicists are competent in all of these methods, 
and since there are wasteful redundancies among them, having a single geometrical lingua franca for physics and other sciences needing rigorous treatment of geometrical topics is highly advantageous. Hestenes lists some of the highlights: ${ }^{7}$

“(1) GA seamlessly integrates the properties of vectors and complex numbers to enable a completely coordinate-free treatment of $2 \mathrm{D}$ physics.

(2) GA articulates seamlessly with standard vector algebra to enable easy contact with standard literature and mathematical methods.

(3) GA Reduces "grad, div, curl and all that" to a single vector derivative that, among other things, combines the standard set of four Maxwell equations into a single equation and provides new methods to solve it.

(4) The GA formulation of spinors facilitates the treatment of rotations and rotational dynamics in both classical and quantum mechanics without coordinates or matrices.

(5) GA provides fresh insights into the geometric structure of quantum mechanics with implications for its physical interpretation.

All of this generalizes smoothly to a completely coordinate-free language for spacetime physics and general relativity."

Nevertheless, as Hestenes points out with more than a little chagrin in his Oersted Lecture, to champion GA is to risk marginalisation or worse in the academic community, since there is a natural inertia in teaching methods to physicists and others, which could only be overcome if GA were to be taught across the undergraduate and graduate curriculum. The need for students to acquire a broad range of techniques in order to read existing treatments means there will be opposition to teaching them what is (wrongly) perceived as "just another" formalism. It is this human and institutional feature that is most likely to hold GA back, despite its many merits. Fortunately Hestenes' enthusiasm has caught in other quarters, most notably among a group of physicists and engineers based in Cambridge, England, called the Cambridge University Geometric Algebra Group. ${ }^{8}$ The existence of such a group is the first indication that Hestenes' battle for the recognition and adoption of GA is becoming less lonely.

Nevertheless it is not inconceivable that we are in for a repeat of the kind of intellectual "war" that saw vectors oust quaternions. As an outsider I am fortunately free of the obligation to adopt or reject the new medium, or to persuade my colleagues to sign up, though by the same token relatively unqualified to judge whether it has intrinsic flaws that have yet to be revealed. So leaving prophesy to others, I pass swiftly to philosophy, where we can be interested in such matters "in principle" and do not need the world to agree.

${ }^{7}$ Quote taken from the online version of the Oersted Lecture at: URL $=<$ http:// modelingnts.la.asu.edu/pdf/OerstedMedalLecture.pdf $>$, p. 2. Retrieved 31.10.2008.

${ }^{8} \mathrm{URL}=<$ http://www.mrao.cam.ac.uk/\%7Eclifford/index.html >. Cf. the textbook Doran and Lasenby (2003), which emanates from the group. 


\section{Philosophical significance of geometric algebra}

Over the long course of interaction between philosophy and mathematics, the philosophers have tended to pay much more attention to pure than applied mathematics. There is a tradition of affinity between philosophy and pure mathematics going back to Plato. It takes in Descartes, Leibniz, Bolzano, Cantor, Frege, Hilbert, Russell and Quine, to name only some of the most prominent. The need for mathematical theories to be in principle applicable is usually held to be easily satisfiable, shown by pointing out how often real and complex analysis are actually used, and how capacious set theory to is represent all manner of structures, mathematical and real. From this perspective the squabbles between quaternionists and vectorists or the advantages of geometric algebra over the hotch-potch of theories that have come to be standardly applied in physics and engineering are disputes beneath the philosopher's attention threshold, to be qualified as merely pragmatic choices, less theoretically exciting than incompleteness, consistency, decidability or large cardinals.

However, while some streams of modern philosophy of mathematics continue in the same vein, since Eugene Wigner asked why mathematics is so "unreasonably effective" in physics (Wigner 1967) and Hartry Field put forward his bold and controversial view that science could in principle do its work without mathematics (Field 1980), the balance has shifted quite agreeably towards looking in closer detail at how mathematics is is in fact applied. ${ }^{9}$ One of the perennial puzzles about mathematics is how it comes about that it is so successful in real-world applications. We are only at the threshold of getting seriously to grips with this problem. As Paolo Mancosu writes, "We badly need more detailed case studies in order to understand better the variety of explanatory uses that mathematics can play in empirical contexts". ${ }^{10}$ The question of applicability is best considered by looking at the mathematics that actually gets applied, which typically is neither set theory nor the sort of complication thrown up by Gödel numbering, but precisely such day-to-day tools as vector analysis.

It is a matter of some depth and intricacy as to how and why one approach to vectors and their kin - the piecemeal approach adopted since 1900 - is or is not better than the integrated approach of geometric algebra. What the case of geometric algebra suggests is that while there can be more than one mathematical formalism or set of techniques which can be applied, which ones in fact are applied may sometimes turn not on effectiveness but on tradition, entrenchment and institutions. On the assumption that geometric algebra is indeed in some sense preferable to the congeries of methods hitherto available, we might wish to

9 See Baker (2005), Bangu (2008), Batterman (2002), Colyvan (2001), French (2000), Lyon and Colyvan (2007), Mancosu (2008), Melia (2000), Steiner (1998 and 2005).

${ }_{10}$ Mancosu (2008). 
consider why this is so. One reason is of course the greater unification and simplicity afforded by geometric algebra over the patchwork approach. But another is whether geometric algebra somehow affords us deeper insight into the nature of space and spacetime, or extension, as Grassmann and Whitehead would have said. That crucial question needs more consideration than it has enjoyed hitherto.

To see how the arguments might go for geometric algebra, let us look at a related but slightly more familiar puzzle: the applicability of complex numbers. ${ }^{11}$ Real numbers and real analysis have so many and such varied applications that their applicability is a yardstick rather than a puzzle. The applications turn to be sure on the many species of quantity, species of attributes admitting of comparison and ratio, and these species fall into a wide variety of more extensive families, ordered by numerous similarities. For example, spatial distances, masses, temporal distances, densities, and the many rates of change all get metrized by real numbers, yet they are different from one another in many ways. By contrast, complex numbers have remarkably few areas of application. The main ones are electrical circuit theory, relativity, and quantum physics. In electrical theory they were introduced by Carl Proteus Steinmetz to enable calculations to be done with AC circuits as easily as using Ohm's Law in DC circuits. ${ }^{12}$ Brilliant as Steinmetz's application is, it is dispensable. Its value lies in its algorithmic simplification. But why does it work at all? The answer lies in the sinusoidal variation of current in AC. The sine and cosine functions are periodic in the reals, whereas the exponential function is positive monotonic. Over the complex numbers the exponential function $e^{z}$ is periodic, and is intimately linked with the others via the well-known equivalence:

$$
e^{x+i y}=\cos x+i \sin y
$$

The occurrence of phases and phase-differences in periodic phenomena, their ready representation by functions of virtual (not real) angles, and the integral treatment of these functions in the complex exponential, gives complex numbers their traction in alternating current calculations. Note that this variation is not connected with real angles in space at all, but in the variation in time of quantities whose equations of variation use functions originally defined in terms of properties of angles.

11 This case prompts Steiner (1998) to the theological conclusion that the universe is "user-friendly": a naturalistic explanation such as that suggested here removes the need for theology. So while I do not agree with Steiner's conclusions, he is asking the right kind of questions.

12 See Kline (1992). 
The application in quantum theory has a different rationale. Quantum theory whether non-relativistic or relativistic uses the mathematics of wavelike phenomena, in the non-relativistic case Schrödinger's or Pauli's wave equations. Again this involves non-spatial periodicity of variation, and phase difference is a crucial feature. This was not peculiar to the quantum level however: wave equations govern electromagnetic phenomena treated classically. What appears different about the quantum case from a mathematical (not physical) point of view is that the variables have to be complex rather than real. It is usual for physicists, when asked why this is so, to shrug their shoulders and say it just is. That is an inherently unsatisfying answer for a philosopher of nature. She wants to know why it is so, what there is about the world in virtue of which only complex functions work at all in this case.

To the modest extent that there is any discussion about this problem, ${ }^{13}$ there is no consensus about why there should be a need for complex numbers. The argument, such as it is one, is principally that they make the results come out right. Yet in any actual observation we only ever observe, record or measure real quantities, and these are always expressed by the real numbers or a real quantity (such as a vector or tensor) parametrized by or defined over the real numbers. The doubling in degrees of freedom brought in by the imaginary parts of the complex quantities used is simply a book-keeping role, rather than one corresponding to features of the world. It would be intellectually preferable if there were a more physically robust account of why the arithmetic needs to work out that way.

While it is still early days to judge, there is a developing interpretation of quantum theory that eliminates complex numbers in favour of real quantities in geometric algebra. Unsurprisingly, it is due to GA's most fervent proponent, David Hestenes. Hestenes suggests that various quantum phenomena including spin, fermion mass, and Heisenberg uncertainty, may be due to a phenomenon postulated in 1930 by Erwin Schrödinger. ${ }^{14}$ The phenomenon is called Zitterbewegung (German for "trembling motion"). Investigating the Dirac equation for a free electron moving in a vacuum, Schrödinger suggested the electron may be moving in a small rapid circular motion whose geometric properties call in GA's bivectors for their expression. Because bivectors are geometrical objects which under the geometric product square to negative real numbers, the arithmetical properties of electrons which in the Schrödinger theory require this feature and so were expressed using complex numbers appear to be expressible purely geometrically, and the puzzle about the otherwise unmotivated introduction of complex numbers disappears.

${ }^{13}$ See the interesting debate between Mark Colyvan (2002) and Joseph Melia (2002) on how we should take the use of complex numbers in applied mathematics.

14 Schrödinger (1930, 1931). See Hestenes (1990). 
Whether such new, geometrically motivated, interpretations of the formalisms of quantum theory will always result in the elimination of complex scalars, remains to be seen. The auspices so far are promising. Speculating ahead, we might raise the following highly desirable prospect for the philosophy of the application of mathematics: a mathematical description of natural phenomena which fully integrates geometrical quantities in such a way as to show that whereas complex quantities are dispensable, real quantities, including geometrical quantities, are not. That would be a consummation devoutly to be wished.*

\section{REFERENCES}

Altmann, S. 1986, Quaternions, Rotations and Double Groups, Oxford: Oxford University Press.

Altmann, S. 1989, 'Hamilton, Rodrigues, and the Quaternion Scandal', Mathematics Magazine 62, pp. 291-308.

Altmann, S. 1992, Icons and Symmetry, Oxford: Oxford University Press.

Altmann, S. and Ortiz, E. (eds.) 2005, Mathematics and Social Utopias in France: Olinde Rodrigues and his Times, Providence: American Mathematical Society.

BaKer, A. 2005, 'Are there Genuine Mathematical Explanations of Physical Phenomena?', Mind 114, pp. 223-238.

Bangu, S. I. 2008, 'Inference to the Best Explanation and Mathematical Realism', Synthese 160, pp. 13-20.

Batterman, R. 2002, The Devil in the Details. Asymptotic Reasoning in Explanation, Reduction, and Emergence, Oxford: Oxford University Press.

Clifford, W. K. 1878, 'Applications of Grassmann's Extensive Algebra', American Journal of Mathematics 1, pp. 350-358.

ClifFord, W. K. 1878 and 1887, Elements of Dynamic: an Introduction to the Study of Motion and Rest in Solid and Fluid Bodies, 2 vols., London: Macmillan.

Clifford, W. K. 1882, 'On the Classification of Geometrical Algebras', in: R. Tucker, ed., Mathematical Papers, London: Macmillan, pp. 397-401.

Colyvan, M. 2001, The Indispensability of Mathematics, Oxford: Oxford University Press.

Colyvan, M. 2002, 'Mathematics and Aesthetic Considerations in Science', Mind 11, pp. 69-78.

Crowe, M. J. 1967, A History of Vector Analysis: The Evolution of the Idea of a Vectorial System, Notre Dame: University of Notre Dame Press.

Doran, C. and Lasenby, A. 2003, Geometric Algebra for Physicists, Cambridge: Cambridge University Press.

Field, H. H. 1980, Science without Numbers. A Defence of Nominalism, Oxford: Blackwell.

French, S. 2000, 'The Reasonable Effectiveness of Mathematics: Partial Stuctures and the Application of Group Theory to Physics', Synthese 125, pp. 103-120.

Grassmann, H. G. 1840, Theorie der Ebbe und Flut (dissertation), reprinted in Grassmann (18941911), Vol. 3.

Grassmann, H. G. 1844, Die lineale Ausdehnungslehre, ein neuer Zweig der Mathematik dargestellt and durch Anwendungen auf die ubrigen Zweige der Mathematik, wie auch auf der Statik, Mechanik, die Lehre vom Magnetismus und die Krystallonomie erläutert, Leipzig: Wigand (reprinted 1878), reprinted in Grassmann (1894-1911), Vol. 1.

Grassmann, H. G. 1862, Die Ausdehnungslehre: vollständig und in strenger Form bearbeitet, Berlin: Enslin, reprinted in Grassmann (1894-1911), Vol. 1.

* My thanks go to two anonymous referees of dialectica for saving me from even greater howlers than those likely to remain.

(C) 2010 The Author. Journal compilation (C) 2010 Editorial Board of dialectica. 
Grassmann, H. G. 1894-1911, Gesammelte mathematische und physikalische Werke, in 3 vols., F. Engel, ed., Leipzig: Teubner, reprinted 1972, New York: Johnson.

Grassmann, H. G. 1995, A New Branch of Mathematics. The Ausdehnungslehre of 1844 and Other Works, trans. by L. C. Kannenberg, Chicago: Open Court.

Hestenes, D. 1966, Space-Time Algebra, New York: Gordon and Breach.

Hestenes, D. 1986, New Foundations for Classical Mechanics, Dordrecht: Kluwer, second edition 1999.

Hestenes, D. 1990, ‘The Zitterbewegung Interpretation of Quantum Mechanics'. Foundations of Physics 20, pp. 1213-1232.

Hestenes, D. 2003, Oersted Lecture 2002: 'Reforming the Mathematical Language of Physics', American Journal of Physics 71, pp. 104-121.

Hestenes, D. and Soвczyк, G. 1984, Clifford Algebra to Geometric Calculus: a Unified Language for Mathematics and Physics, Dordrecht: Reidel.

KLINE, R. R. 1992, Steinmetz: Engineer and Socialist, Baltimore: Johns Hopkins University Press.

Kuipers, J. P. 2002, Quaternions and Rotation Sequences: A Primer with Applications to Orbits, Aerospace and Virtual Reality, Princeton: Princeton University Press.

Lyon, A. and M. Colyvan 2007, 'The Explanatory Power of Phase Spaces', Philosophia Mathematica 16, pp. 227-243.

Mancosu, P. 2008, 'Explanation in Mathematics', in: E. N. Zalta, ed., The Stanford Encyclopedia of Philosophy (Fall 2008 Edition), URL=<http://plato.stanford.edu/archives/fall2008/entries/ mathematics-explanation/>.

Melia, J. 2000, 'Weaseling Away the Indispensability Argument', Mind 109, pp. 455-479.

Melia, J. 2002, Response to Colyvan, Mind 111, pp. 75-79.

SCHRÖDINGER, E. 1930, 'Über die kräftefreie Bewegung in der relativistischen Quantenmechanik', Berliner Berichte, pp. 418-428.

SCHRÖDINGER, E. 1931, 'Zur Quantendynamik des Elektrons', Berliner Berichte, pp. 63-72.

SteIner, M. 1998, The Applicability of Mathematics as a Philosophical Problem, Cambridge MA: Harvard University Press.

Steiner, M. 2005, 'Mathematics - Applications and Applicability', in: S. Shapiro, ed., The Oxford Handbook of Philosophy of Mathematics and Logic, Oxford: Oxford University Press, pp. 625-650.

Whitehead, A. N. 1898, A Treatise on Universal Algebra, with Applications, Cambridge: Cambridge University Press, reprinted New York: Hafner, 1960.

Wigner, E. 1967, 'The Unreasonable Effectiveness of Mathematics in the Natural Sciences', in: Symmetries and Reflections, Bloomington: Indiana University Press, pp. 222-237. 